\title{
On the Performance of Multi-Gateway LoRaWAN Deployments: An Experimental Study
}

\author{
Konstantin Mikhaylov*†, Martin Stusek*, Pavel Masek*, Radek Fujdiak*‡, Radek Mozny*, \\ Sergey Andreev ${ }^{\S}$, and Jiri Hosek* \\ *Department of Telecommunications, Brno University of Technology, Brno, Czech Republic \\ ${ }^{\dagger}$ Center for Wireless Communications, University of Oulu, Oulu, Finland \\ $\ddagger V S B$ - Technical University of Ostrava, Ostrava, Czech Republic \\ ${ }^{\S}$ Faculty of Information Technology and Communication Sciences, Tampere University, Tampere, Finland
}

\begin{abstract}
A remarkable progress in the Low Power Wide Area Network (LPWAN) technologies over the recent years opens new opportunities for developing versatile massive Internet of Things (IoT) applications. In this paper, we focus on one of the most popular LPWAN technologies operating in the license-exempt frequency bands, named LoRaWAN. The key contribution of this study is our unique set of results obtained during an extensive measurement campaign conducted in the city of Brno, Czech Republic. During a three-months-period, the connectivity of a public Long Range Wide Area Network (LoRaWAN) with more than 20 gateways (GWs) was assessed at 231 test locations. This paper presents an analysis of the obtained results, aimed at capturing the effects related to the spatial diversity of the GW locations and the real-life multi-GW network operation with all its practical features. One of our findings is the fact that only for $47 \%$ tested locations the GW featuring the minimum geographical distance demonstrated the highest received signal strength and signal-to-noise ratio (SNR). Also, our results captured and characterized the variations in the received signal strength indicator (RSSI) and SNR as a function of the communication distance in an urban environment, and illustrated the distribution of the spreading factors (SFs) as a result of the adaptive data rate (ADR) algorithm operation in a real-life multi-GW deployment.
\end{abstract}

Index Terms-LPWAN, LoRaWAN, spatial diversity, multiple gateways, experiment, performance, measurement

\section{INTRODUCTION}

Every single year both the number and the utility of the miniature machines deployed around us increase dramatically. The data invisibly flowing through the air between the various sensors, servers, data processing systems, and actuators become an essential enabler for the novel and diverse Internet of Things (IoT) applications. Substantial efforts have been invested over the past years to develop efficient wireless connectivity solutions to serve the massive IoT [1] to improve the continuity of these data flows.

Among the diverse technologies composing the IoT connectivity landscape, the group of solutions, which are jointly referred to as Low Power Wide Area Networks (LPWAN) [1], [2], showed tremendous growth in the past few years [3]. The ultimate aim of these technologies is to provide a scalable, cost- and energy-efficient option for the non-critical IoT applications, subject to infrequent transmissions of small data chunks by each device. To achieve this, many of the LPWAN technologies utilize star-of-stars topology and aim to maximize the last-hop wireless communication distance.

The LPWAN technologies available today can be divided into two major groups. The first one consists of the 3rd Generation Partnership Project (3GPP) Narrow-Band IoT (NBIoT) and Long-Term Evolution-Machine (LTE-M) radios. The aforementioned solutions have evolved from the Long-Term Evolution (LTE) and are intended to operate in the licensed frequency bands. For this reason, they emphasize the efficient use of valuable time-frequency resources, thus improving the communication reliability at the cost of more advanced and energy-hungry signaling.

Another group is composed of multiple LPWAN technologies operating in the available-for-everyone unlicensed frequency bands. Given that these bands are shared by various non-synchronized systems, some of which are not employing any listen-before-talk provisioning, the interference is often inevitable. For this reason, the LPWAN technologies operating in the unlicensed bands typically use simpler, often Alohabased, channel access mechanisms. Saving on signaling and thus reducing the related energy consumption, this introduces a challenge of improving the chances of packet delivery. This challenge is often addressed through either, or a combination of, the following four mechanisms: (i) increase of the transmission time and/or signal spreading, (ii) repetition of a packet, (iii) use of error-correcting codes, and (iv) employment of receiver spatial diversity.

Two of the unlicensed-bands LPWAN technologies currently dominate the market: Sigfox and Long Range Wide Area Network (LoRaWAN) [3]. The Sigfox solution is based on the use of ultra-narrow band radio signal and supports only one modulation-coding scheme (MCS) for the uplink channel. The business model resembles that of the traditional cellular telecom, but there is a single operator deploying multiple gateways in each country. The LoRaWAN solution uses more broadband signals and supports multiple MCSs allowing to achieve different trade-offs between the on-air time and the maximum communication range by modifying the spreading factor (SF) parameter of the LoRa modulation [4]. When it comes to the business model, the LoRaWAN supports not only operator-deployed public networks but also applicationor user-specific private networks. 


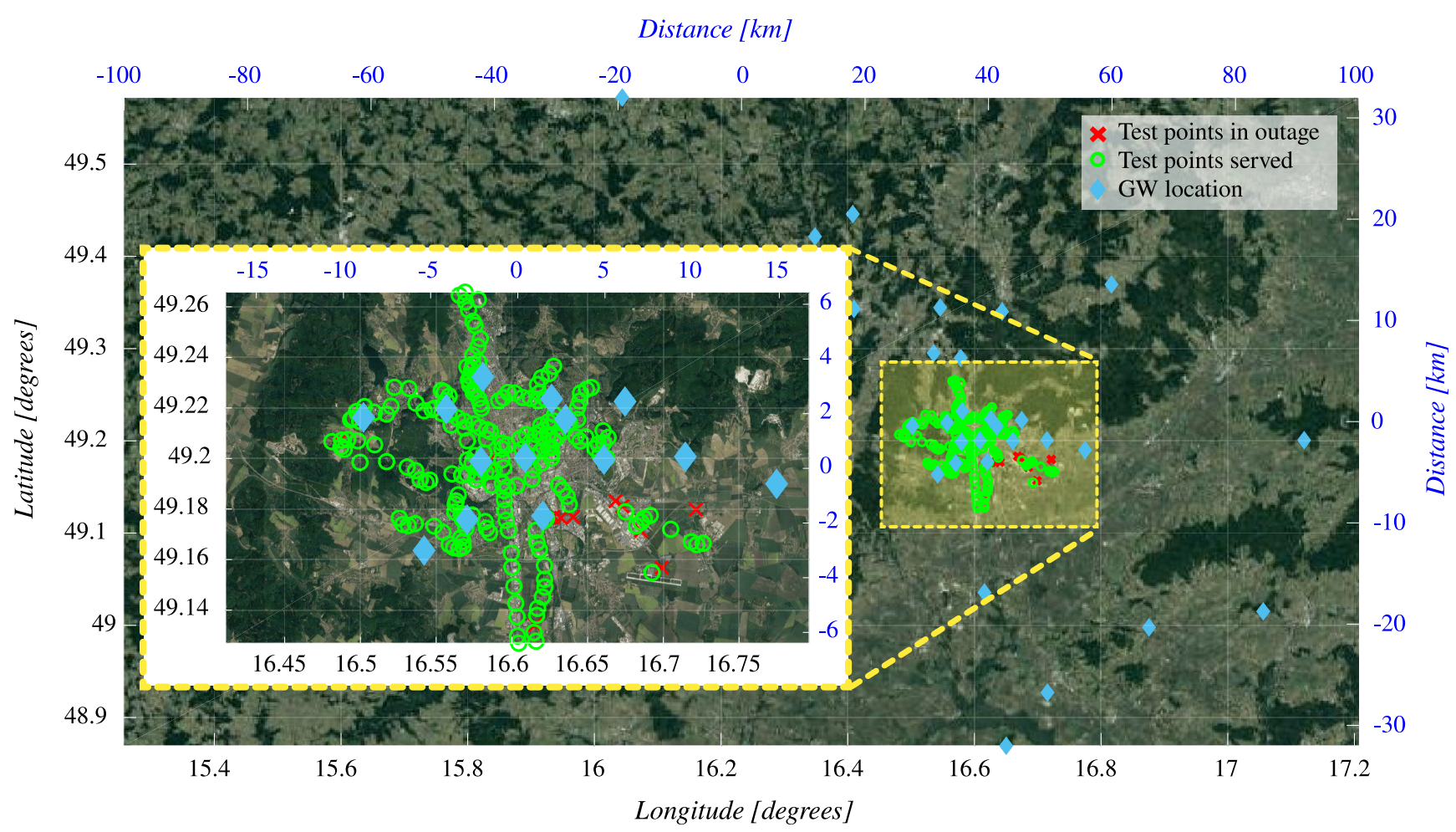

Fig. 1. Map of the test area with the locations of the GWs and served/outaged test locations.

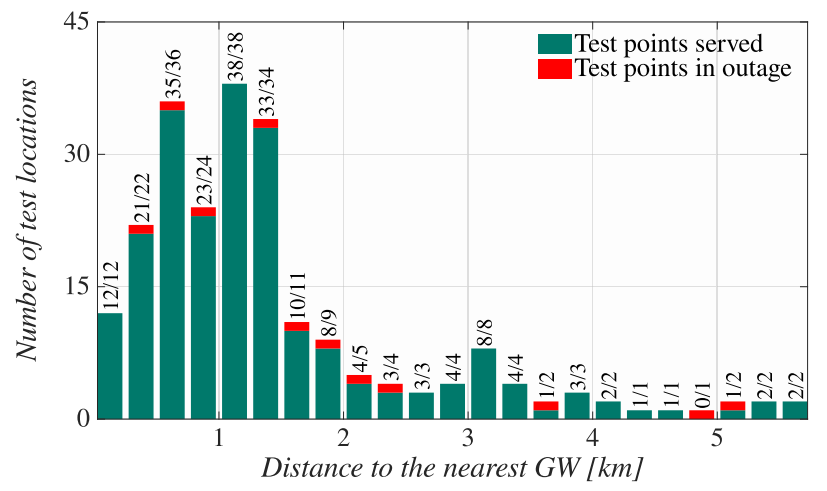

Fig. 2. Effect of the distance to the nearest GW on the outage probability.

Both LoRaWAN and Sigfox have been in active commercial deployment since late 2015 and are currently present in multiple countries around the globe. In the past years, these technologies have also been actively addressed by the academia. The LoRaWAN, owing to the ease of a private network deployment, has been experimentally studied in detail. Specifically, by now, multiple papers discuss the coverage of a LoRaWAN network in various geographical regions [5][10]. Typically, these measurements are limited to only a few dozens of test points. Another usual limitation in these studies is the use of only a single gateway (GW). Meanwhile, spatial diversity is one of the critical mechanisms for improving the scalability and reliability of LoRaWAN technology [11], [12].

To address this omission, in this paper, we report the results of an extensive measurement campaign conducted during three months in the city of Brno, Czech Republic. During this cam- paign, we have tested the radio connectivity from more than two hundred of different outdoor test locations to a commercial public LoRaWAN network with more than twenty GWs. In what follows, we report the results of our experimental data analysis, by showing the real-life performance of a large scale multi-GW LoRaWAN network and revealing several interesting effects in such a deployment. These findings and the results of their analysis constitute the major contribution of this paper.

The rest of this text is organized as follows. In Section II, we review the relevant technical background information about the architecture and the operation of LoRaWAN. In Section III, we detail our experimental setup and procedures, and in Section IV we report and discuss our key results. Finally, Section V concludes the paper and communicates its main messages.

\section{LORAWAN TECHNOLOGY BACKGROUND}

Each LoRaWAN network includes three different types of devices: the end devices (EDs), one or several GWs, and a network server (NS) [14]. Additionally, a network may contain a specialized join server (JS) to handle and coordinate internetwork roaming. Typically, the EDs can send their data at any time using an Aloha-like channel access mechanism. For their uplink transmission, the EDs randomly select one of up to sixteen frequency channels supported by the network (e.g., in $868 \mathrm{MHz}$ Industrial, Scientific, and Medical (ISM) band in Europe and $915 \mathrm{MHz}$ ISM band in the US). Both the transmit power and the SF (which, for the operation in the EU region, can take up to 6 different values ranging from 
SF7 to SF12 [13]) can be adjusted based on the radio channel conditions for each ED. With an increase of the SF by one the on-air time of the packet increases 1.5-2 times, while 1.5-2 $\mathrm{dB}$ are added to the potential link budget. Due to the need for saving the energy, the EDs are typically configured to use the smallest SF permitting reliable communication. This is achieved either through a manual definition of the SF to use, or through the adaptive data rate (ADR) mechanism [14]. The signals with different SFs are quasi-orthogonal, and the GWs are typically designed to support simultaneous reception of the signals in every possible frequency channel with each possible SF. Each of the GWs uses a backbone IP connection to deliver all the received and decoded packets to the NS, which discards the packet duplicates, stores the received packets in a database, and may forward them to the dedicated application servers (ASs). As one can see, in contrast to the conventional cellular networks, in LoRaWAN networks the EDs are not associated to a particular GW.

To enable downlink communication, following each uplink transmission, an ED opens up to two receive windows (RWs) within the pre-specified time slots. The RW1 is opened in the same frequency channel and typically uses the SF utilized for uplink. If nothing has been received in RW1, the EDs should open another RW (i.e., RW2) in a pre-specified channel using a pre-defined SF. These RWs can be used by the NS to deliver an acknowledgment, provide certain application data, or carry commands. In addition to these RWs, which are obligatory for all EDs (devices having only these RWs are reffered to as class A EDs), the EDs may optionally feature additional periodic RWs (class B EDs) or spend all their idle time receiving (class C EDs) [14].

\section{Measurement Setup and Procedure}

The experimental measurements reported in this paper were conducted from February to April 2019 around the city of Brno, Czech Republic. The test area spans over $12 \mathrm{~km}$ north to south and $24 \mathrm{~km}$ west to east. In total, the measurements were conducted in 231 various outdoor test locations ${ }^{1}$. As the test points, we have selected the stop stations of public transport (i.e., buses, trams, and trolleybuses) as it stands for one of the potential use-case - localization services for LoRaWAN in Smart City applications. The test area is served by more than twenty different LoRaWAN GWs, belonging to the network of one of the Czech telecom operators, which employs the $868 \mathrm{MHz}$ band. Note that when the measurements were planned and conducted the locations of the GWs were yet unknown to the authors and thus no optimization of the experiment point locations with respect to the GW position was handled. All the transmissions were done in the three obligatory for the EU LoRaWAN frequency channels (i.e., $868.1,868.3$, and $868.5 \mathrm{MHz}$ ) using the default coding rate (i.e., 4/5).

During the measurements, we employed a commercial LoRaWAN test device (i.e., the ARF8123A [15]) with a GPS

\footnotetext{
${ }^{1}$ The map of measurement points in question is accessible online from the Google Maps at http://tiny.cc/tsfejz
}

receiver onboard. The test device was configured with all the parameters to enable its activation by personalization (ABP). To obtain the maximum coverage, the test device was configured to use only the maximum SF (i.e., SF12) and transmit power (i.e., $14 \mathrm{dBm}$ ). The models and the characteristics of the GWs are unknown to us.

The measurement procedure was as follows. The class A test device was moved to a selected test point and positioned with the dipole antenna pointing towards the sky at a distance of approximately one meter from the ground, away from any buildings, walls, or other obstacles. Then the device was powered up to transmit five uplink packets. The timeframes for the experiments and the accurate location of each test point were recorded. Note that the measurements in different regions were done during different days within the specified time period. The measurements were performed during the office hours (10 a.m. to 4 p.m.) on week days.

After the end of the experiments, all the data (including the timestamps, RSSI, SNR, and location of the GW) were downloaded from the LoRaWAN NS and machine-processed by the MATLAB scripts. Specifically, each data entry was mapped to a specific test location, the geographical distance from each test location to the GWs was calculated, and all the measurements were statistically processed. Note that unlike a conventional LoRaWAN NS, which typically deletes the packet duplicates received from different GWs, the one used in our experiments was configured to keep all the duplicates. This enabled us to obtain a unique insight into how spatial diversity affects the performance of a real-life multi-GW LoRaWAN.

\section{Measurement Results}

First, we analyze from which of the test points at least one radio packet has been received. The map of the test area and the positions of the nearby LoRaWAN GWs are shown in Fig. 1. In red we marked the test locations having an outage, i.e., the ones, from which no packets have been received. There were only 12 such locations out of 231 tested in total (thus giving the overall outage probability of about $5.2 \%$ ). The cumulative packet delivery rate (PDR) throughout the entire experiment campaign was $83 \%$. This level of PDR is sufficient for some delay- and loss-tolerant IoT applications, but is well below the needs of dependable IoT use cases. Note that these results have been obtained for the case where neither packet acknowledgements nor retransmissions were employed.

Further, we examine the probability of a test location being in outage depending on its distance to the nearest GW. For this, we calculate the geographical distance between each test point and the nearest GW, and, based on that, distribute all test locations into 23 bins having the width of 250 meters. These results are illustrated in Fig. 2. As one can see, the maximum distance from a test location to the nearest $\mathrm{GW}$ in all our experiments was below $8 \mathrm{~km}$, with $40 \%$ of the tested points located within $1 \mathrm{~km}$ range, and $80 \%$ - within $2 \mathrm{~km}$. Interestingly, half of the test points from which no packets were delivered, are located within $2 \mathrm{~km}$ range of the $\mathrm{GW}$, including one of them located within $500 \mathrm{~m}$ range. Given the 


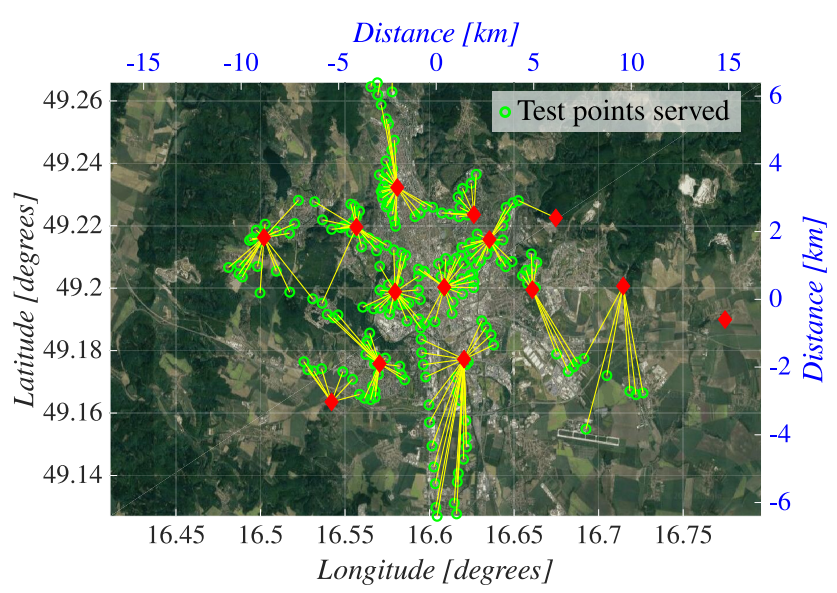

(a) The geographically-closest GWs

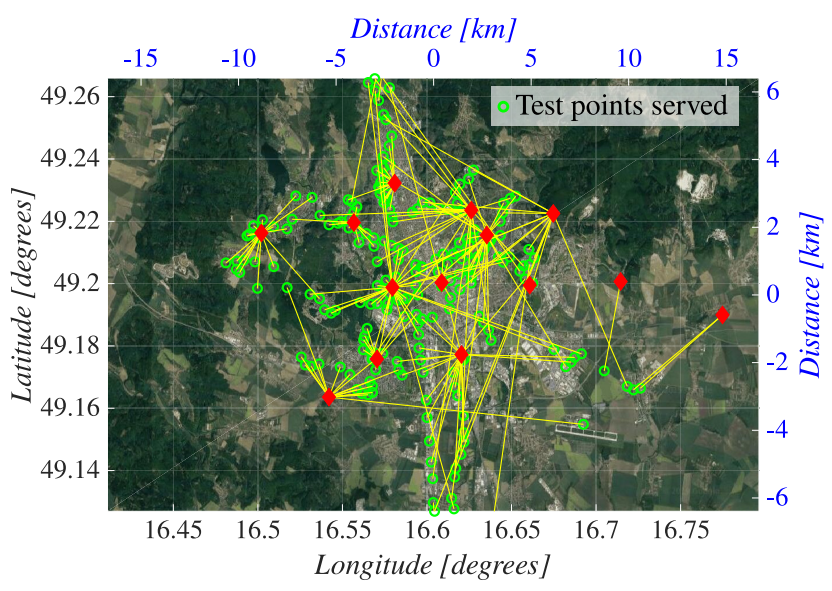

(b) The GWs featuring highest RSSI

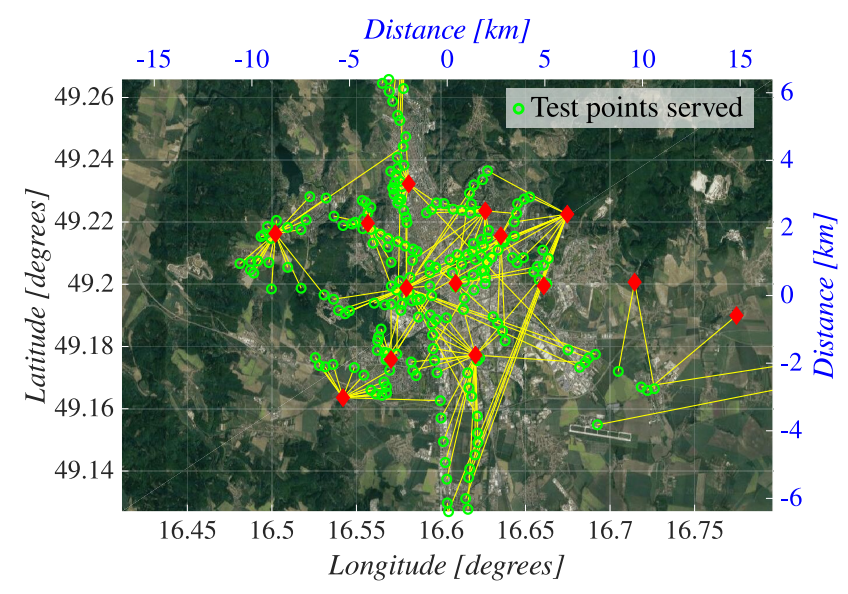

(c) The GWs featuring highest SNR

Fig. 3. The GW with best performance for each test location.

city environment with the presence of buildings blocking the line-of-sight (LOS) link, this result is hardly surprising.

For the test locations not in outage, we determine the statistics for the number of GWs receiving each packet. Specifically, the mean number of GWs receiving each packet was 4.17 and the standard deviation was 2.77. Also, we have observed that the same packet has been received by at maximum 15

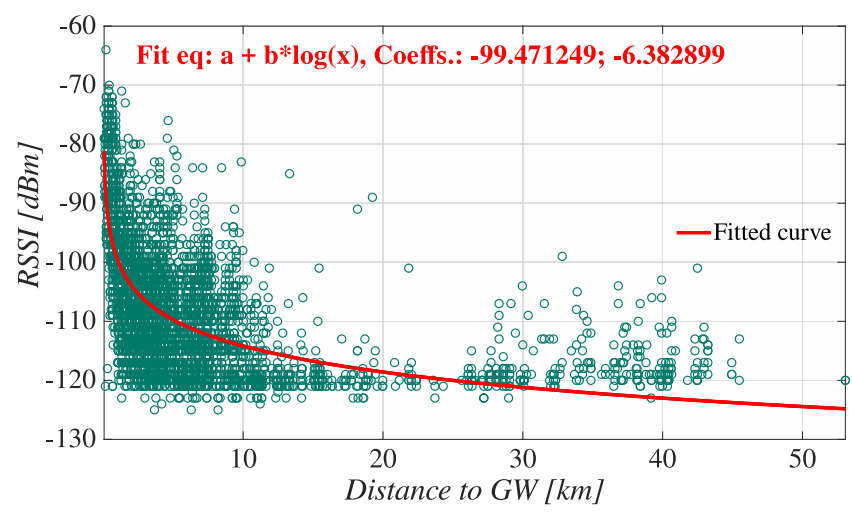

Fig. 4. RSSI vs. distance for each received radio packet.

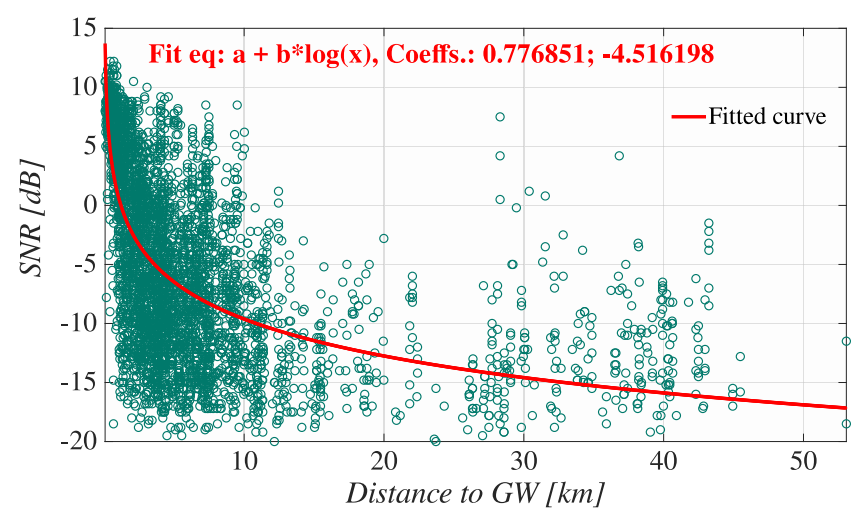

Fig. 5. SNR vs. distance for each received radio packet.

GWs. Furthermore, we analyzed for each test location which of the GWs was the closest one to the test location, and which featured the highest RSSI and SNR. These results are illustrated in Figs. 3a, 3b, and 3c, respectively. As one can see, often (in $45 \%$ of the cases), the nearest GW was not featuring the highest RSSI, or (in $40 \%$ of the cases) the highest SNR. Moreover, rather often (34\% of the cases), the best SNR and RSSI were demonstrated by different GWs. Finally, in only $47 \%$ of the cases, the GW closest to a test location featured both the highest RSSI and SNR.

Furthermore, Figs. 4 and 5 demonstrate the effect of the communication distance between a test location and a GW on the RSSI and SNR, respectively, for each correctly received radio packet during our experiments. The least-squares fit, which demonstrates the dependencies and its form are presented on the charts as well. Interestingly, the graphs clarify that during our trials several packets were received from a distance exceeding $50 \mathrm{~km}$, and quite a few - within the range of 30 to $45 \mathrm{~km}$. Another intriguing result is the reported RSSI limits: -64 to $-125 \mathrm{dBm}$. Typically, the sensitivity of a LoRaWAN transceiver operating with SF12 is expected to be about $-137 \mathrm{dBm}$, but we have never observed any values below $-127 \mathrm{dBm}$. The SNR threshold for SF12, according to [16], is $-20 \mathrm{~dB}$, which matches decently well the minimum value observed in our experiments. The dynamic range of the SNR 


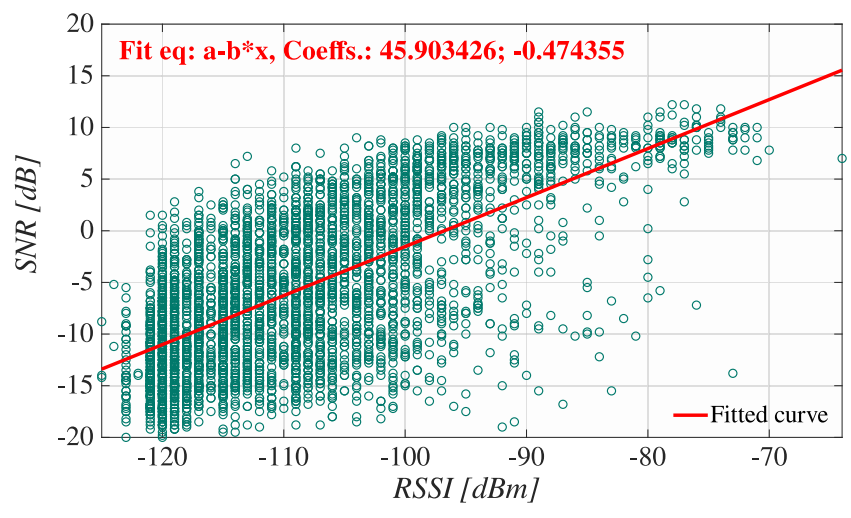

Fig. 6. SNR vs. RSSI for each received radio packet.

TABLE I

SF ASSIGNMENT BY ADR

\begin{tabular}{|c|c|c|c|c|c|c|}
\hline \multirow{2}{*}{ Method } & \multicolumn{6}{|c|}{ Number of test locations with a particular SF } \\
\cline { 2 - 7 } & SF7 & SF8 & SF9 & SF10 & SF11 & SF12 \\
\hline RSSI-based & 219 & 0 & 0 & 0 & 0 & 0 \\
\hline SNR-based & 0 & 33 & 84 & 58 & 32 & 12 \\
\hline
\end{tabular}

recorded during our measurements was about $34 \mathrm{~dB}$. Finally, Fig. 6 depicts the interrelation between the RSSI and the SNR.

Despite the fact that for both charts the major trends confirm intuition - with the increase in the communication distance both the RSSI and the SNR degrade - there are several effects, which are worth noting. First, there is a high deviation of both the RSSI and the SNR of the signals received from the test points located within the range of between 0.5 to about $10 \mathrm{~km}$ distance from the GW. We expect this to be caused by the non-line-of-sight (NLOS) propagation for some radio signals. For moderately short communication distances, even the packets suffering from refractions may still be received and successfully decoded by a GW. Meanwhile, with an increase in the communication distance, only the packets sent from the test locations in the direct LOS carry enough power to be correctly decoded. This can also explain the resulting shape of the RSSI versus SNR curve.

Finally, using our experimentally measured radio channel characteristics, we produce observations on how the SFs will be distributed between all our test locations by the ADR algorithm. In the state-of-the-art literature, the two different approaches for SF distribution are dominating. The first one is based on the RSSI and the sensitivity threshold of the radio receiver and has been proposed in [17]. This approach is often implied in analytical works. The other one, which is also implemented by the real-life commercial LoRaWAN GWs (e.g., [18]) and detailed further in the Semtech application note [16], is based on the SNR. Note that for the SNR-based algorithm, inline with [16], we implied that with a decrease of the SF by one, the SNR degrades by $2.5 \mathrm{~dB}$. The RSSI thresholds were derived from Table 1 in [17]. The results are depicted in Figs. 7 and 8, respectively. Furthermore, the number of the test locations experiencing each particular SF are listed in Table I.

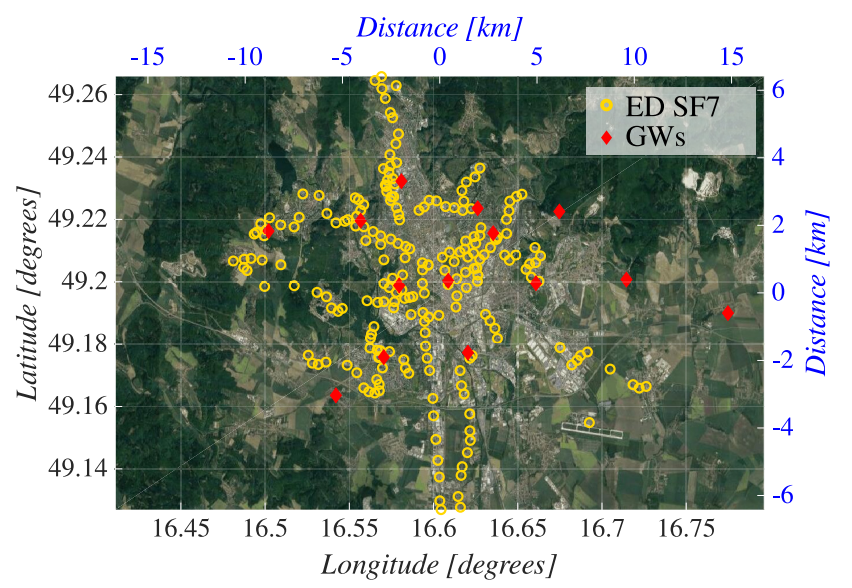

Fig. 7. SF allocation based on RSSI and sensitivity.

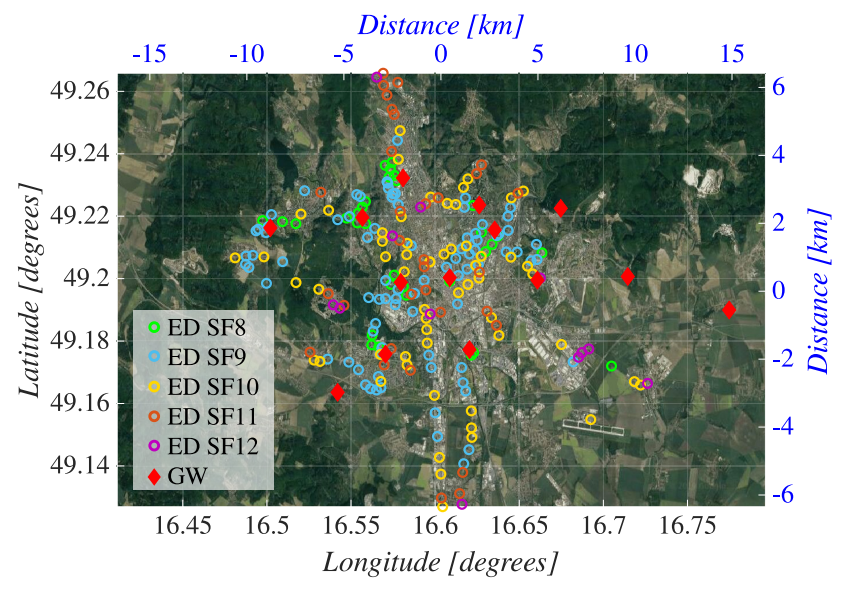

Fig. 8. SF allocation based on SNR.

As one can see, the results from the two algorithms are dissimilar. According to [17], the sensitivity of a LoRa radio receiver for SF7 is on the order of $-126.5 \mathrm{dBm}$, which is lower than the real-life RSSI values measured in our experiments. Using the algorithm from [16], the majority of the test locations would be served by SF9 and SF10.

\section{Discussion And Conclusions}

In this paper, we reported the results of a unique and extensive measurement campaign focused on the real-life performance of a public LoRaWAN deployment composed of multiple gateways. The measurements were carried out in 231 different outdoor locations on the streets of the city of Brno, Czech Republic. Even though the experimental results are dependent on the network setup and are hard to generalize, they shed some light on the operation and the performance of a real-life multi-GW LoRaWAN deployment with all its complexity. For this reason, the presented results can be of interest to many stakeholders, including the network planners, the application designers, and the academia, for whom they provide a ground-truth reference and point out the relevant real-life challenges to be addressed. In what follows, we summarize our key results and notable observations. 
- Despite using the highest SF and transmit power, and operating in an area covered by multiple GWs, from several test locations we have not received even a single packet. However, from more than $94 \%$ of the test locations, at least one packet has been received. The total PDR during the entire measurement campaign was $83 \%$.

- Analyzing the distribution of the GWs and the test locations, we can see that for each test location there has been at least one GW within $8 \mathrm{~km}$ range. For $80 \%$ of the test locations, a $\mathrm{GW}$ was present within $2 \mathrm{~km}$ range.

- Each uplink packet has been received on average by 4 GWs and at maximum by 15 GWs. The maximum communication distance we observed exceeded $50 \mathrm{~km}$, but the majority of the links have been much shorter.

- In only 55\% of the cases, the closest GW featured the highest RSSI and in only 60\% cases - the highest SNR.

- The difference between the SNR/RSSI for the test locations at the same distance from a GW can be very significant, especially at a distance on the order of kilometers.

- The conventional SNR-based ADR mechanism assigns SF9 (38\% points) or SF10 (26\% points) for the majority of the tested locations. SF11 and SF8 would be allocated for about $15 \%$ of the locations each. Only about $5 \%$ of the locations will have to be served with SF12.

It is very likely that the specifics of Brno natural and urban landscape - the presence of hills and mid-rise building - was one of the key factors obstructing the radio communication and affecting the obtained results. Our analysis of the results also revealed that the received packets have been distributed between the three frequency channels in use rather uniformly (33.5\% vs. $32.8 \%$ vs. $33.7 \%$ ). Hence, given such a uniform use of the channels, none of them was affected by the interference much stronger than the others. Nonetheless, one cannot be certain that there have been no broadband interference or interference from other EDs, or other (e.g., private) LoRaWAN networks about which we are not aware and which were operating on the same frequency channels. It is also worth noting that all our test locations were outdoors. Therefore, should one wish to deploy the sensors indoors, the variations of the performance might have been even more significant.

The results of our experiments demonstrate that some of the common assumptions regarding the communication in LoRaWAN networks - the radio channel behavior, the locations of the serving GWs, the distribution of SFs between the nodes - do not hold tight in practice. This fact calls for the development of more accurate models to be used in simulations and analytical studies. Further, the limited number of measurements and their limited time span did not allow us to capture all aspects of temporal behaviour of the channel (e.g., shadowing and channel fading) - these have to be addressed as a part of a more extensive measurement campaign. Another perspective direction of further contributions is the optimization of the network planning and configuration, as well as development of new channel access mechanisms for a multi-GW environment.

\section{ACKNOWLEDGMENT}

The described research was supported by the National Sustainability Program under grant LO1401. For the research, the infrastructure of the SIX Center was used. This article is based upon support of international mobility project MeMoV, No. CZ.02.2.69/0.0/0.0/16_027/00083710 funded by European Union, Ministry of Education, Youth and Sports, Czech Republic and Brno University of Technology. This research has been supported by Academy of Finland 6G Flagship (grant 318927), project RADIANT, LPWAN-evolution project, and project 5G-FORCE.

\section{REFERENCES}

[1] G. A. Akpakwu, B. J. Silva, G. P. Hancke, and A. M. Abu-Mahfouz, "A Survey on 5G Networks for the IoT: Communication Technologies and Challenges," IEEE Access, vol. 6, pp. 3619-3647, 2018.

[2] U. Raza, P. Kulkarni, and M. Sooriyabandara, "Low Power Wide Area Networks: An Overview," IEEE Commun. Surv. \& Tut., vol. 19, no. 2, pp. 855-873, Secondquarter 2017.

[3] J. Blackman, "LPWA matchup - LoRaWAN vs Sigfox vs NB-IoT vs LTE-M: trading blows (round 3)", available: http://tiny.cc/lufejz

[4] M. Chiani and A. Elzanaty, "On the LoRa Modulation for IoT: Waveform Properties and Spectral Analysis," IEEE IoT J., vol. 6, no. 5, pp. 8463-8470, Oct. 2019.

[5] R. El Chall, S. Lahoud, and M. El Helou, "LoRaWAN Network: Radio Propagation Models and Performance Evaluation in Various Environments in Lebanon," IEEE IoT J., vol. 6, no. 2, pp. 2366-2378, Apr. 2019.

[6] E. Harinda, S. Hosseinzadeh, H. Larijani, and R. M. Gibson, "Comparative Performance Analysis of Empirical Propagation Models for LoRaWAN $868 \mathrm{MHz}$ in an Urban Scenario," in Proc. 5th IEEE World Forum IoT, Limerick, 2019, pp. 154-159.

[7] F. J. Grin, G. O. Petracca, D. F. Lipuma, and E. R. Amig, ”LoRa network coverage evaluation in urban and densely urban enviroment simulation and validation tests in Autonomous City of Buenos Aires," in Proc. XVII Workshop Inf. Process. and Control, Mar del Plata, 2017, pp. 1-5.

[8] M. M. Erbati, G. Schiele, and G. Batke, "Analysis of LoRaWAN technology in an Outdoor and an Indoor Scenario in Duisburg-Germany," in Proc. Int. Conf. Comp. Commun. Syst., Nagoya, 2018, pp. 273-277.

[9] J. Petajajarvi, K. Mikhaylov, A. Roivainen, T. Hanninen, and M. Pettissalo, "On the coverage of LPWANs: range evaluation and channel attenuation model for LoRa technology," in Proc. 14th Int. Conf. ITS Telecommun., Copenhagen, 2015, pp. 55-59.

[10] J. Petajajarvi, K. Mikhaylov, R. Yasmin, M. Hamalainen, and J. Iinatti, "Evaluation of LoRa LPWAN Technology for Indoor Remote Health and Wellbeing Monitoring", Int. J. Wireless Inf. Netw., vol. 24, no. 2, pp. 153-165, June 2017.

[11] M. Ni, M. Jafarizadeh, and R. Zheng, "On the Effect of Multi-Packet Reception on Redundant Gateways in LoRAWANs," in Proc. IEEE Int. Conf. Commun., Shanghai, 2019, pp. 1-6.

[12] F. Van den Abeele, J. Haxhibeqiri, I. Moerman, and J. Hoebeke, "Scalability Analysis of Large-Scale LoRaWAN Networks in ns-3," IEEE IoT J., vol. 4, no. 6, pp. 2186-2198, Dec. 2017.

[13] G. Ferre and A. Giremus, "LoRa Physical Layer Principle and Performance Analysis," in Proc. 25th IEEE Int. Conf. on Electron., Circ. and Syst., Bordeaux, 2018, pp. 65-68.

[14] N. Sornin (ed.), LoRaWAN 1.1 Specification, October 2017.

[15] ARF8123A: Field Test Device LoRaWAN EU863-870, available: https://www.adeunis.com/en/produit/ftd-868-915-2/

[16] Semtech, LoRaWAN simple rate adaptation recommended algorithm, Revision 1.0, Oct. 2016.

[17] F. Cuomo, M. Campo, A. Caponi, G. Bianchi, G. Rossini, and P. Pisani, "EXPLoRa: Extending the performance of LoRa by suitable spreading factor allocations," in Proc. 13th IEEE Int. Conf. Wireless and Mobile Comput., Networking and Communs., Rome, 2017, pp. 1-8.

[18] Conduit AEP - LoRaWAN Upgrade guide, available: http://tiny.cc/i3cejz 